\title{
Empathic responses in clinical practice: Intuition or tuition?
}

\author{
Robert Buckman MD PhD, James A. Tulsky MD, Gary Rodin MD
}

$\mathrm{D}$ uring recent interviews for admission to medical school, candidates were asked to respond to a hypothetical situation so that their communication skills could be assessed. They were told that a patient in the emergency department had just been informed that he had suffered a minor heart attack, to which he had exclaimed "Oh no! A heart attack! That's terrible!" They were then asked to provide an empathic response to the patient's distress, one that clearly identified and acknowledged at least one of the emotions the patient had expressed. Most of the candidates responded appropriately with statements such as "I realize that's a big shock to you" or "Clearly that news feels overwhelming." However, one candidate replied "I did say it was a small heart attack, you know."

\section{What is empathy?}

Although the last candidate's response was factual, it neglected to acknowledge the patient's emotional state. Whereas the candidate's clarification might have been helpful at some other point in the course of treatment, it was unlikely to build rapport or reassure at that particular instant. It would certainly not have scored highly on any scale measuring clinical empathy.

Empathy is the ability to understand another's experience, to communicate and confirm that understanding with the other person and to then act in a helpful manner. ${ }^{1}$ Despite some overlap with other compassionate responses, particularly sympathy, empathy is distinct. ${ }^{2}$ Sympathy involves the sharing of emotions between the observer and the sufferer without necessarily disentangling the experience of the former from the latter. By contrast, empathy is an accurate understanding of the experience of the sufferer leading to an attuned response from the observer. ${ }^{3}$

Clinical empathy is an essential element of quality care, and is associated with improved patient satisfaction and adherence to treatment, as well as fewer malpractice complaints. ${ }^{4}$ Indeed, many of the criticisms of medical care voiced by patients refer to what they perceive as inadequate interpersonal and communication skills rather than deficiencies in the technical or procedural aspects of their care. We suggest that an increase in the attention paid by physicians to their own role in this interaction could mitigate the distress that they feel during particularly difficult encounters with patients and may possibly improve their satisfaction with the practice of medicine. There is even evidence to suggest the involvement of a neurobiologic substrate in this exchange. Functional magnetic resonance imaging data indicate that acts of kindness toward another person may activate important reward centres in one's own brain, whereas unsympathetic communication can elicit negative emotions. ${ }^{5}$ These findings imply that explicit articulation of empathy and other altruistic behaviours may benefit both donor and recipient.

\section{How often do physicians engage in empathic communication?}

In clinical practice, physicians articulate explicit responses to their patients' emotions infrequently. Take, for example, a recent study ${ }^{6}$ in which oncologists were video-recorded speaking with their patients. Moments when emotions were expressed by the patients (e.g., statements such as "I've got nothing to look forward to.") were often overlooked by the physicians. Of approximately 200 such moments, termed empathic opportunities, the oncologists responded $22 \%$ of the time. They chose instead to discuss some other aspect of medical care, such as a change in therapy, $76 \%$ of the time. A more recent study involving patients with lung cancer and their oncologists showed similar results. ${ }^{7}$ In this study, the oncologists acknowledged or explored only $11 \%$ of such empathic opportunities.
Competing interests: Robert Buckman regularly lectures on communication skills and often receives an honorarium for this work from such organizations as Cancer Care Ontario, Million Dollar Round Table and the Lexington Forum.

No competing interests

declared by the other authors.

This article has been peer reviewed.

Correspondence to: Dr. Robert Buckman, robert.buckman@uhn.on.ca

CMAJ 2011.DOI:10.1503 /cmaj.090113

\section{KEY POINTS}

- Clinical empathy is an essential element of quality care associated with improved patient satisfaction and adherence to treatment, as well as with fewer malpractice complaints.

- There is abundant evidence that empathic communication is a skill that can be taught.

- Currently, there is insufficient emphasis and time apportioned to teaching the empathic response in medical school, postgraduate training and continuing medical education. 


\section{What accounts for the skill deficit in clinical empathy?}

There are likely multiple explanations for the relatively low levels of empathic communication shown in these studies. Until recently, education concerning communication and clinical empathy skills lagged behind education in other areas of clinical practice. As a result, many physicians have not received sufficient training to develop or enhance the necessary interpersonal skills for patient-centred care - a model identified as ideal by the US Institute of Medicine. ${ }^{8}$ It is also possible that empathy is not adequately modelled, nor is its acquisition adequately rewarded or reinforced, during the education process. Finally, systematic education in this area may have developed slowly because acquiring competence in these domains was considered to be a mysterious and osmotic process (i.e., an intuition) rather than a skill set that can be specified, taught and learned (i.e., a technique that would lend itself to tuition).

\section{Can empathy be taught?}

There is now abundant evidence that empathic communication is an essential medical skill that can be taught. ${ }^{3}$ Even though physicians differ in their innate capacities, as with any other skill in medicine, clinical empathy can be taught and acquired. Improvements have been seen at every level of training and practice. In fact, the medical school candidate mentioned earlier offered an excellent empathic response on her second attempt after less than 15 seconds of further instruction - a clear demonstration of the effectiveness of teaching clinical empathy. Therefore, it is not surprising that changes in medical school

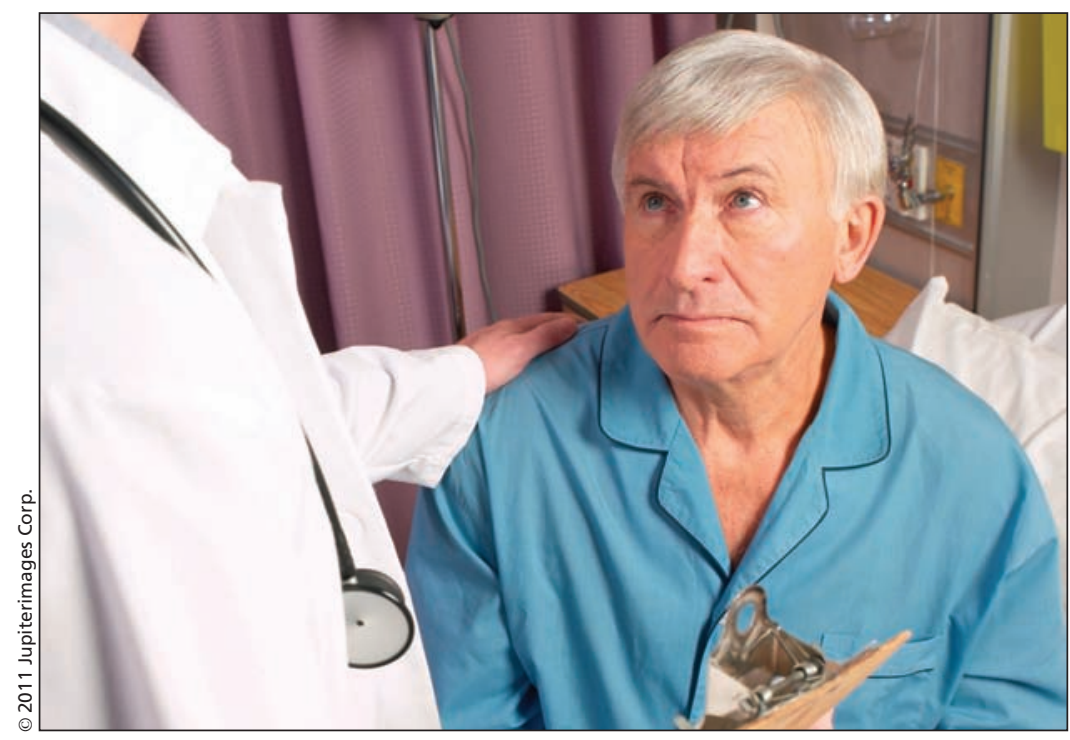

curricula to include emotion skills training have shown encouraging results. ${ }^{910}$ Resident physicians attending short courses have improved their ability to deliver bad news, specifically developing better responses to emotional cues, ${ }^{11}$ and fellows in oncology attending workshops on communication skills showed substantial improvements in verbal expressions of empathy. ${ }^{12}$

The recent development of valid and reliable measures to assess clinical empathy, such as the Consultation and Relational Empathy (CARE) measure,${ }^{13}$ has facilitated the teaching and evaluation of these skills, as have the objective structured clinical examination (OSCE) stations. ${ }^{14} \mathrm{Sim}-$ ilar methods can be used to change the behaviour of practising physicians. In a randomized controlled study of communication skills training that included 160 oncologists from 34 UK cancer centres, those who received the training showed significantly more expressions of empathy and appropriate responses to patient cues in videorecorded encounters than their untrained counterparts. ${ }^{15} \mathrm{~A}$ more recent study has shown that similar improvements in the use of empathic responses can be achieved even when oncologists are trained using a brief online format that solicits feedback on audio-recorded encounters. ${ }^{16}$

\section{Where do we go from here?}

Clinical empathy is an essential medical skill that can be taught and improved, thereby producing changes in physician behaviour and patient outcomes. Empathic responsiveness, which conveys understanding of another's perspective ${ }^{17}$ requires the integration of knowledge, skills and attitudes that should be taught during undergraduate medical training and continue in a more contextualized fashion at the postgraduate level and beyond. Despite evidence that empathy may be related to clinical competence ${ }^{18}$ and that it declines during undergraduate medical training, ${ }^{19}$ medical schools in Canada and elsewhere may not be adequately addressing the issue at the undergraduate and postgraduate levels. There is little information concerning the specific teaching of clinical empathy as a distinct technique; when it is taught, it is usually included in the curriculum covering communication skills. Clearly, more emphasis on empathic communication in medical education in Canada and other countries would be valuable and would benefit from further study.

Our profession now needs to incorporate the teaching of clinical empathy more widely into clinical practice at all levels beginning with the selection of candidates for medical school. The behavioural aspects of empathy - the empathic response - can be assessed and integrated into 
medical schools' core communication skills training. Assessment of such responses not only provides a reward for their occurrence, it also delineates their value as an integral part of becoming a competent physician. Consonant with the principles of adult learning,$^{20}$ the relevance of empathic skills must also be reinforced and contextualized throughout medical education.

Although formal assessment of clinical empathy and inclusion of empathy training in medical school curricula is a good start, its relevance must be contextually reinforced and embedded in postgraduate and continuing medical education (CME) programs. A voluntary CME program on empathy for physicians may help the individual practitioners who pursue it gain general knowledge and skills in this area, but there is no guarantee that those who need it most will seek it out. Moreover, a separate CME course for empathy risks decontextualizing the acquisition of communication skills from clinical practice. Wider use can be made of single half-day teaching sessions (as a minimum) comprising brief didactic presentations, followed by supervised role play using standardized patients and DVD- or Web-based examples to help integrate the skills into clinical practice. ${ }^{21}$ Ultimately, it may be possible to design a Web-based quiz for recertification of clinicians in practice in which different responses and their consequences are illustrated on video, as in the award-winning Crossroads ${ }^{22}$ section of the I*CARE website. In Canada, the Royal College of Physicians and Surgeons' Maintenance of Certification (MOC) Program and other programs of, for example, the College of Family Physicians of Canada, could reinforce the value of empathy by including patients' evaluations of physicians' empathic skills, which can be assessed within the broader framework of communication skills.

The maintenance of empathic skills requires more than just targeted educational interventions. It also requires a shift in the learning environment such that clinicians practise in ways that reinforce and model the importance of empathy in patient care..$^{23}$ The question of what specific educational interventions are needed to enhance empathic skills at all levels of education must be addressed by medical educators, as must the socalled hidden curriculum by which attitudes and values are implicitly communicated to trainees. To borrow from the words of Winston Churchill, we have the tools - now we can finish the job.

\section{References}

1. Mercer SW, Reynolds WJ. Empathy and quality of care. $\mathrm{Br} J$ Gen Pract 2002;52(Suppl):S9-12.

2. Stepien KA, Baernstein A. Educating for empathy: a review. $J$ Gen Intern Med 2006;21:524-30.

3. Neumann M, Bensing J, Mercer S, et al. Analyzing the "nature" and "specific effectiveness" of clinical empathy: a theoretical overview and contribution towards a theory-based research agenda. Patient Educ Couns 2009;74:339-46.

4. Levinson W, Roter D, Mullooly JP, et al. Physician-patient communication: the relationship with malpractice claims among primary care physicians and surgeons. JAMA 1997;277:553-9.

5. Moll J, Krueger F, Zahn R, et al. Human fronto-mesolimbic networks guide decisions about charitable donation. Proc Nat Acad Sci USA 2006;103:15623-8.

6. Pollak KI, Arnold RM, Jeffreys AS, et al. Oncologist communication about emotion during visits with patients with advanced cancer. J Clin Oncol 2007;25:5748-52.

7. Morse DS, Edwardsen EA, Gordon HS. Missed opportunities for interval empathy in lung cancer communication. Arch Intern Med 2008; 168:1853-8.

8. Institute of Medicine, Committee on Quality of Health Care in America. Crossing the quality chasm: a new health system for the 21st century. Washington (DC): The National Academies Press; 2001.

9. Hook KM, Pfeiffer CA. Impact of a new curriculum on medical students' interpersonal and interviewing skills. Med Educ 2007; 41:154-9.

10. Satterfield JM, Hughes E. Emotion skills for medical students: a systematic review. Med Educ 2007;41:935-41.

11. Back AL, Arnold RM, Baile WF, et al. Efficacy of communication skills training for giving bad news and transitions to palliative care. Arch Intern Med 2006;167:453-60.

12. Alexander SC, Keitz SA, Sloane R, et al. A controlled trial of a short course to improve residents' communication with patients at the end of life. Acad Med 2006;81:1008-12.

13. Mercer SW, Maxwell M, Heaney D, et al. The consultation and relational empathy (CARE) measure: development and preliminary validation and reliability of an empathy-based consultation process measure. Fam Pract 2004;21:699-705.

14. Eva KW, Rosenfeld J, Reiter HI, et al. An admissions OSCE: the multiple mini-interview. Med Educ 2004;38:314-26.

15. Fallowfield L, Jenkins V, Farewell V, et al. Efficacy of a Cancer Research UK communication skills training model for oncologists: a randomised controlled trial. Lancet 2002;359:650-6.

16. Tulsky JA, Alexander SC, Olsen MK, et al. Can oncologists be taught to respond to patients' expressions of negative emotions? Results of the SCOPE Trial [presentation]. American Academy on Communication in Healthcare Research and Teaching Forum; 2008 Oct. 17-19; Madison (WI).

17. Mercer SW, Reynolds WJ. Empathy and the quality of care. $\mathrm{Br}$ $J$ Gen Pract 2002;52:S9-13.

18. Hojat M, Gonnella JS, Mangione S, et al. Empathy in medical students as related to clinical competence, gender and academic performance. Med Educ 2002;36:522-7.

19. Hojat M, Mangione S, Nasca TJ, et al. An empirical study of the decline in empathy in medical school. Med Educ 2004;38:934-41.

20. Knowles MS, Holton EF, Swanson RA. The adult learner. 6th ed. Philadelphia (PA): Elsevier; 2005.

21. Buckman R. Practical plans for difficult conversations in medicine. Baltimore (MD): Johns Hopkins University Press; 2010.

22. MD Anderson Cancer Center. Crossroads. Houston (TX): The Center; 2010. Available: www.mdanderson.org/education-and -research/resources-for-professionals/professional-educational -resources/i-care/complete-library-of-communication-videos /crossroads.html (accessed 2010 Dec. 2).

23. Spencer J. Decline in empathy in medical education: how can we stop the rot? Med Educ 2004;38:916-8.

Affiliations: From the Department of Medical Oncology and Hematology, Princess Margaret Hospital and the Faculty of Medicine (Buckman), University of Toronto, Toronto, Ont.; the Center for Palliative Care and the Department of Medicine (Tulsky), Duke University, Durham VA Medical Center, Durham, NC, and the Department of Psychosocial Oncology and Palliative Care, Princess Margaret Hospital and the Department of Psychiatry (Rodin), University of Toronto, Toronto, Ont.

Contributors: Robert Buckman and Gary Rodin conceived of and cowrote most of the manuscript. James Tulsky authored most of the section "Can empathy be taught?" and reviewed many drafts of the manuscript and the references. All of the authors approved the version submitted for publication.

Acknowledgement: The authors are extremely grateful to Dr. Adrienne Tan for discussions, reviews of the published literature and constructive comments on many topics raised in this article. 